Recebido em 11/2019. Aceito para publicação em 12/2019.

\title{
COMPÓSITOS DE PET, PÓS-CONSUMO, COM MICA
}

\section{POST-CONSUMPTION PET AND MICA COMPOSITES}

\author{
Jéssica Fernandes Pereira ${ }^{1}$ \\ Monique Cristina Carvalho Mattos ${ }^{2}$ \\ Florêncio Gomes de Ramos Filho ${ }^{3}$ \\ Shirleny Fontes Santos ${ }^{4}$
}

Resumo: O aumento do consumo das embalagens de bebidas feitas de PET, trouxe consigo o problema do acúmulo de resíduos pós-consumo no meio ambiente. De modo que a demanda por meios de reuso desses materiais é crescente. Uma alternativa é a obtenção de compósitos com cargas inorgânicas, como a mica. Neste contexto, o presente trabalho teve como objetivo produzir um compósito baseado em poli (tereftalato de etileno) e mica (muscovita). Utilizou-se a mica com granulometria na faixa de $-45 \mu m$. Para o processamento das misturas de PET/mica em extrusora monorrosca, a mica foi adicionada nas proporções de 3, 5 e 7\%, em seguida os compósitos foram prensados para obtenção de filmes planos e as amostras foram caracterizadas. Nos testes de dureza Shore $D$ e densidade, observou-se ligeiro aumento com aumento do teor de mica. O índice de Fluidez (MFI) também aumentou para maiores concentrações de mica, o que pode estar relacionado ao efeito lubrificante apresentado pela muscovita. Em relação às análises térmicas, na termogravimetria (TG) foi observado apenas um evento de perda de massa na faixa de temperatura entre 350 e $500^{\circ} \mathrm{C}$ correspondente à decomposição do polímero. Já na análise de DSC, foi possível observar um pico endotérmico, a cerca de $250^{\circ} \mathrm{C}$ referente à fusão do $P E T$. Por meio das análises de microscopia foi possível observar as partículas de mica dispersas na matriz polimérica.

Palavras-chave: Compósito; reciclagem; muscovita; poli(tereftalato de etileno).

Abstract: The increased consumption of beverage packaging made of PET has brought with it the problem of post-consumer waste accumulation in the environment. Thus, the demand for means of reuse of these materials is increasing. An alternative is to obtain composites with inorganic fillers, such as mica. In this context, the present work aimed to produce a composite based on poly (ethylene terephthalate) and mica (muscovite). Mica with particle size in the range of $-45 \mu \mathrm{m}$ was used. For the processing of PET/mica mixtures in single-screw extruder, the mica was added in the proportions of 3, 5 and 7\%, then the composites were pressed to obtain films and the samples were characterized. Shore $D$ hardness and density tests showed a slight increase with increasing mica content. The fluidity values (MFI) also increased for higher mica concentrations, which may be related to the lubricating effect of muscovite. In the thermogravimetry analysis (TG), one mass loss event was observed in the temperature range between $350-500^{\circ} \mathrm{C}$, corresponding to polymer decomposition. In the DSC analysis, one endothermic peak was observed, at about $250^{\circ} \mathrm{C}$ for PET fusion. Optical microscopy analysis allowed the observation of the dispersed mica particles in the polymeric matrix.

Keywords: Composite; recycling; muscovite; poly (ethylene terephthalate).

\footnotetext{
1 Graduada em Tecnologia de Polímeros, Centro Universitário Estadual da Zona Oeste, Brasil. E-mail: jessicaf.pereira@yahoo.com.br.

${ }^{2}$ Mestre em Ciência e Tecnologia de Mateirais pelo Centro Universitário Estadual da Zona Oeste, Brasil. E-mail: nique.matos@hotmail.com.

3 Professor Adjunto do Centro Universitário Estadual da Zona Oeste, Brasil. E-mail: florenciogr@yahoo.com.

${ }^{4}$ Professora Adjunta do Centro Universitário Estadual da Zona Oeste, Brasil. E-mail: lenyfontes@yahoo.com.br.
} 


\section{INTRODUÇÂO}

O Poli (tereftalato de etileno), conhecido pela sigla em inglês PET, é classificado quimicamente como um copolímero poliéster obtido pela reação de condensação entre o etilenoglicol e o ácido tereftálico (TPA). Apresenta, entre outras propriedades, excelente estabilidade dimensional, fácil conformação, versatilidade de design e cores, fácil processamento, alta resistência ao impacto e alta reciclabilidade, além da possibilidade de se apresentar no estado amorfo (transparente), parcialmente cristalino e orientado (translúcido) e altamente cristalino (opaco) (TEÓFILO et al., 2014; SANTOS et al, 2018).

Dentre os muitos usos do PET, a aplicação como embalagem para bebidas é uma das mais difundidas e que cresce a cada dia no Brasil e no mundo, porém esse tipo de aplicação faz com que ele apresente vida útil muito curta, e acabe muitas vezes sendo descartado de forma inadequada junto com outros polímeros causando poluição ambiental (ROMÃO; SPINACÉ; DE PAOLI, 2009; FERREIRA; FONSECA; SARON, 2011; SANTOS et al., 2018).

Entretanto, a embalagem de PET pós-consumo, após a reciclagem mecânica, já vem sendo utilizada para a produção de carpetes, tapetes, cordas, cerdas de vassoura, escovas e tecidos não tecidos (TNT), entre outros usos. Usos esses menos nobres do que os da resina virgem devido ao fato que uma das maiores dificuldades nesse tipo de reciclagem é a depreciação das propriedades mecânicas devido a processos de degradação por cisão hidrolítica da cadeia polimérica. A redução da massa molar do polímero, torna-o mais rígido e quebradiço o que torna muitas vezes inviável seu reaproveitamento (FERREIRA; FONSECA; SARON, 2011; BIMESTRE; SARON, 2014).

Uma alternativa promissora para melhorar a qualidade do PET reciclado ocorre por meio da preparação de compósitos, em que, usualmente o PET será a matriz e uma carga será adicionada ao sistema, de modo a conseguir melhoria de propriedades (LEITE et al., 2011).

Especialmente, compósitos baseados na interação entre um polímero e argilominerais como bentonita, montmorilonita e muscovita vêm promovendo desenvolvimento de materiais com propriedades mecânicas e térmicas melhoradas (LEITE et al., 2011; ESCÓCIO et al., 2011; MONSORES et al., 2017; BANDA-CRUZ; FLORES-GALLARDO; RIVERA-ARMENTA, 2017).

Particularmente, a mica do tipo muscovita é um mineral de baixo custo e grande disponibilidade no Brasil. Ela pertence a uma ampla classe dos minerais da família dos filossilicatos do tipo alumino-silicato e é considerada uma carga semi-reforçadora para polímeros orgânicos (ESCÓCIO et al., 2011).

Em geral, as micas apresentam alta rigidez dielétrica, excelente resistência a intempéries, excelente estabilidade química, baixa condutividade térmica, isolamento elétrico, estabilidade em altas temperaturas, características de não inflamabilidade, e 
relativamente baixo custo (BALTAR et al., 2008; RELOSI et al., 2018).

A bibliografia especifica sobre o sistema PET/mica manifestou-se escassa, todavia um dos poucos trabalhos disponíveis na literatura sobre o tema e em língua portuguesa é o trabalho de Sirelli (2008), no qual foram estudados compósitos de poli(tereftalato de etileno) e mica muscovita. Nos resultados, foi verificado que a adição da mica em quantidades superiores a $10 \%$ tornaram o material frágil e que as propriedades mecânicas e o peso molecular diminuíram consideravelmente. Esse estudo também analisou que a adição da mica no compósito diminui a viscosidade do material no estado fundido quando comparado com o PET puro e teve pouco efeito sobre o grau de cristalinidade da matriz polimérica. A adição da mica no PET provocou uma perda de mobilidade das cadeias poliméricas em baixas temperaturas, aumentando a temperatura da transição secundária $\beta$ e também a temperatura de transição vítrea dos compósitos em relação ao PET para todos os percentuais de mica.

No entanto, a mica já vem encontrando largo uso como carga de reforço em outros sistemas poliméricos, como no estudo que tratou da incorporação deste mineral com composições vulcanizáveis de borracha natural. Verificou-se que a adição de até $40 \%$ de mica promoveu melhora de cerca de $300 \%$ no módulo de elasticidade, além de melhoras na dureza, densidade de ligações cruzadas e deformação por compressão. Porém, o melhor desempenho quanto a resistência ao rasgamento e a tensão ruptura foi obtido com $30 \%$ de mica (ESCOCIO et al., 2003).

Dentre muitos dos sistemas poliméricos nos quais a muscovita já vem sendo utilizada, pode-se citar também o estudo realizado por MONSORES, 2017, no qual a introdução da mica muscovita em teores de 5-20\% em matrizes de polipropileno resultou em melhoria das propriedades térmicas e mecânicas do polímero, com o melhor desempenho obtido com o teor de $15 \%$ de muscovita.

Neste contexto, o presente trabalho teve como objetivo desenvolver compósitos baseados na interação entre PET e mica muscovita.

\section{METODOLOGIA}

\subsection{Tratamento da mica}

A mica, tipo muscovita, utilizada neste trabalho é um mineral oriundo da região nordeste do Brasil e foi coletado e beneficiado pelo Centro de Tecnologia Mineral (CETEM) do Rio de Janeiro, Santos, França e Ogasawara (2011). Esta mica, já beneficiada e com tamanho de partícula inferior a $125 \mu \mathrm{m}$, foi moída em moinho de facas laboratorial IKA, e classificada granulometricamente para obter o mineral com granulometria inferior a $45 \mu \mathrm{m}$. 


\subsection{Processamento dos compósitos}

O PET utilizado consistiu de garrafas pós-consumo, as quais foram lavadas e cominuídas por corte manual. Foram preparadas misturas para processamento em extrusora monorosca, CHILLROLL AX PLÁSTICOS, entre PET e concentrações de mica de 3, 5 e $7 \%$ em peso do mineral em granulometria inferior a $45 \mu \mathrm{m}$. As condições de extrusão foram: 260,270 e $285^{\circ} \mathrm{C}$ nas zonas 1,2 e 3 da extrusora, respectivamente e velocidade de extrusão de $34 \mathrm{rpm}$. Após processamento, as amostras foram prensadas para obtenção de filmes planos utilizando prensagem a quente a $250^{\circ} \mathrm{C}$, com pressão de 3 toneladas. A Figura 1 exibe, para exemplificar, fotografia de um dos filmes obtido a partir da amostra PET/3\%Mica.

Figura 1 - Fotografia do filme plano da amostra PET/3\%Mica.

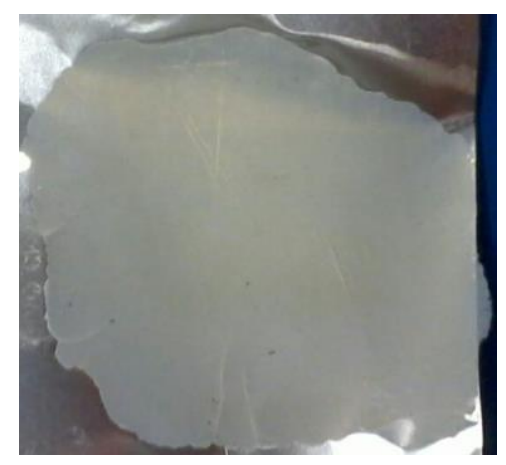

Fonte: Os autores.

\subsection{Técnicas de caracterização}

A estrutura cristalográfica da mica foi determinada por difratometria de raios- $X$, com radiação Co $\mathrm{Ka}(35 \mathrm{kV} / 40 \mathrm{~mA}) ; 2 \theta$ na faixa de 5 a $80^{\circ}$. A morfologia do mineral foi investigada por microscopia eletrônica de varredura (MEV), em equipamento da marca Leica, modelo F440.

Os compósitos foram avaliados quanto a densidade, utilizando densímetro Digital GEHAKA DSL 910, segundo a ASTM D792-08, quanto a dureza Shore D, utilizando Durômetro MEDTEC (ASTM D2240-05) e quanto ao índice de fluidez (ASTM D123810) com uso do Plastômetro MELT SLOW (CEAST). Os compósitos foram também analisados por microscopia óptica de alta capacidade utilizando microscópio óptico da marca OLYMPUS, modelo BX51M.

As análises de TG/DSC foram realizadas em equipamento simultâneo da TA INSTRUMENT modelo Q1000. As condições de análise foram: taxa de aquecimento de $10^{\circ} \mathrm{C} / \mathrm{min}$; faixa de temperatura variando da temperatura ambiente até $700^{\circ} \mathrm{C}$, em atmosfera de nitrogênio. 


\section{Resultados e Discussão}

\subsection{Caracterizações da muscovita}

Realizou-se a análise por difração de raios- $X$ da amostra de mica após moagem em moinho laboratorial, como mostrado na Figura 2. É possível verificar que a estrutura cristalina do mineral não foi alterada e não surgiram picos de outras fases que podem surgir, devido a processos de moagem quando a amostra sofre alguma alteração. Dados da literatura comprovam que o aspecto difratográfico obtido corresponde à estrutura esperada do mineral micáceo muscovita (SANTOS; FRANÇA; OGASAWARA, 2011; MONSORES et al., 2017).

Figura 2 - Difratograma de raios-x da mica muscovita.

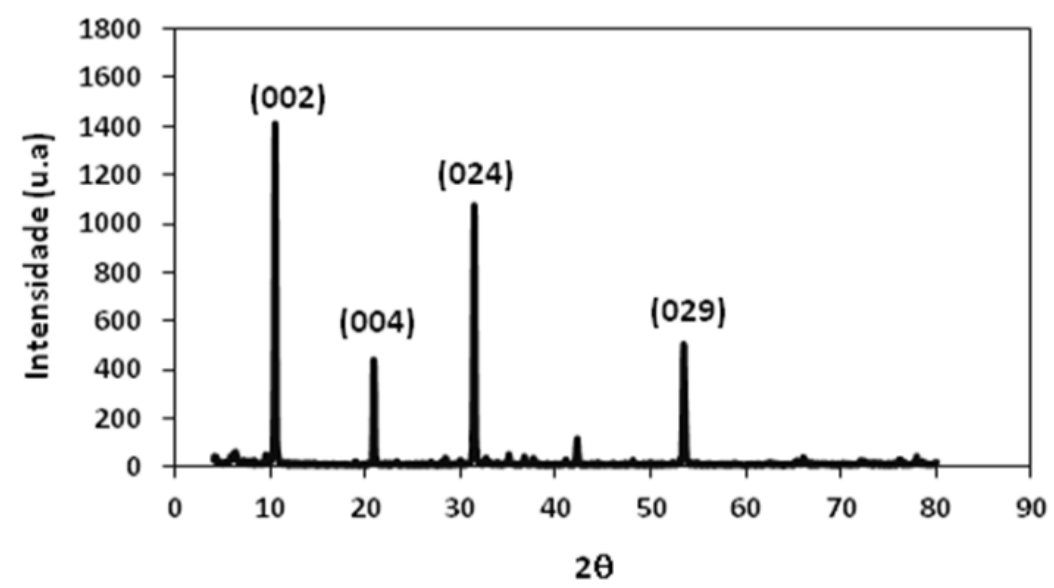

Fonte: Os autores.

Avaliou-se também a morfologia da muscovita, após a moagem, como mostrado na Figura 3, que revelou sua estrutura lamelar típica que não sofreu alteração em função do tratamento realizado. Uma análise qualitativa da imagem também permite verificar que os tamanhos de partículas do mineral também se encontravam na faixa desejada.

Figura 3 - MEV da mica muscovita após moagem.

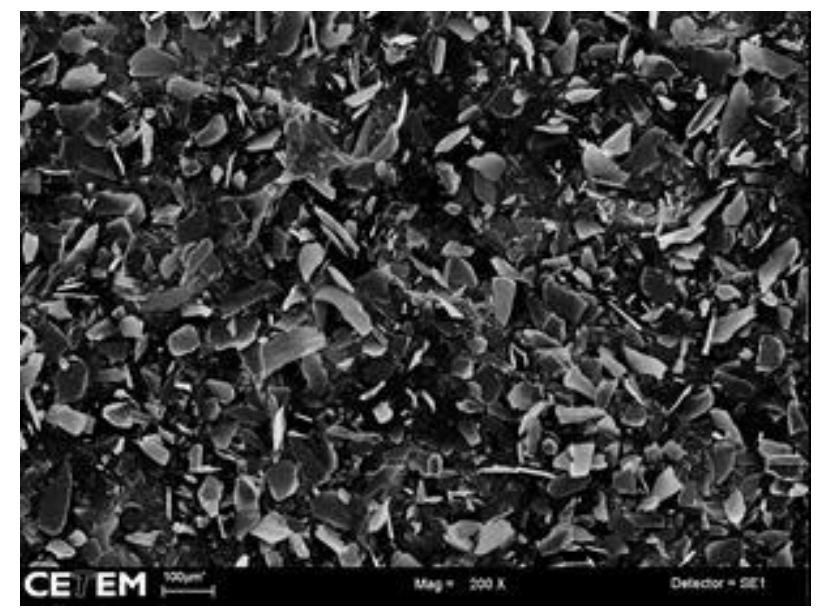

Fonte: Os autores.

\subsection{Caracterizações dos compósitos}


$\mathrm{Na}$ Tabela 1 estão apresentados os valores de densidade, dureza e índice de fluidez das amostras obtidas por meio do processamento da muscovita e do PET pósconsumo.

Tabela 1 - Valores de densidade, dureza e índice de fluidez dos compósitos PET/mica.

\begin{tabular}{cccc}
\hline Compósitos & Densidade $\left(\mathrm{g} / \mathrm{cm}^{3}\right)$ & Dureza (Shore D) & MFI (g/10 mim) \\
\hline PET puro & $0,807 \pm 0,006$ & $78,8 \pm 0,8$ & $11,66 \pm 2,12$ \\
PET/Mica 3\% & $1,498 \pm 0,041$ & $81,8 \pm 0,8$ & $11,66 \pm 2,23$ \\
PET/Mica 5\% & $1,546 \pm 0,001$ & $83,4 \pm 0,9$ & $16,36 \pm 2,63$ \\
PET/Mica 7\% & $1,612 \pm 0,008$ & $84,0 \pm 0,7$ & $22,71 \pm 2,00$ \\
\hline
\end{tabular}

Fonte: Os autores.

A análise dos resultados de densidade apresentados na Tabela 1 mostra que o próprio PET puro após processamento apresenta queda no valor de densidade em relação ao da resina virgem que varia entre 1,3-1,4 g/ $/ \mathrm{cm}^{3}$, entretanto com aumento do teor da mica muscovita, ocorreu um aumento da densidade, para valores próximos e até maiores ao da resina virgem, o que sugere, portanto, a ação reforçadora da mica no sistema (MONSORES et al., 2017; LEITE et al, 2011).

Os resultados de dureza, Tabela 1, apresentam apenas ligeiro aumento da propriedade com aumento do teor do mineral, sugerindo pouca eficiência da mica na melhoria dessa propriedade dentro da faixa de concentração estudada. Trabalhos com sistemas similares evidenciam que esta propriedade geralmente sofre alterações mais significativas para mais altos teores da carga (MONSORES et al, 2017; ALMEIDA, 2018).

O índice de fluidez, Tabela 1, aumentou para os teores de mica de 5 e 7\%. Dados da literatura (MANCINI; BEZERRA; ZANIN, 1988; ROMÃO; SPINACÉ; DE PAOLI, 2009) indicam que um aumento dessa propriedade poderia estar relacionada à diminuição do peso molecular do polímero, em caso de reprocessamento sem uso de extensores de cadeia ou outros aditivos. No caso em questão, há ainda a presença da mica que pode apresentar um efeito lubrificante quando submetida a aquecimento. Para o sistema em questão, acredita-se que uma mistura dos dois fenômenos pode ter causado a diminuição desse índice (MANCINI; BEZERRA; ZANIN, 1998; DU et al, 2018).

Os resultados da análise de termogravimetria (TG) e da Calorimetria exploratória diferencial (DSC) estão exibidos nas Figuras 4 e 5 . Nos perfis térmicos de TG do PET puro e dos compósitos PET/Mica, como mostrado na Figura 4, é possível verificar a presença de apenas um evento de perda de massa na faixa de temperatura entre 350 e $500^{\circ} \mathrm{C}$, valores correspondentes à decomposição do polímero. As curvas do PET puro e dos compósitos apresentaram perfis bastantes similares, não tendo sido possível 
observar influência significativa da introdução da mica no perfil térmico (PEREIRA et al, 2017; GANDARA; GONÇALVES; SARON, 2017).

Figura 4 - Perfis de análise termogravimétrica (TG) para as amostras de PET puro e PET/Mica.

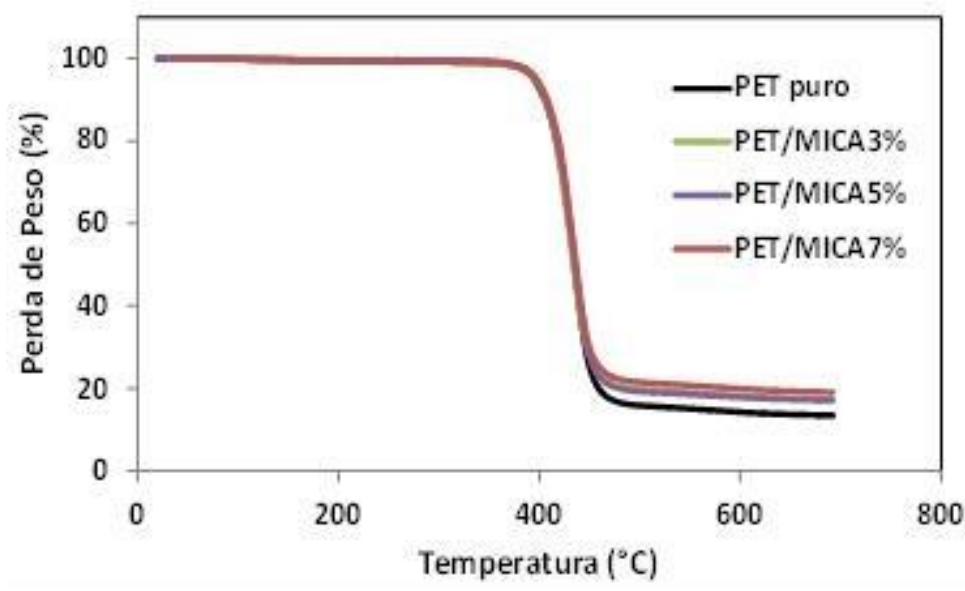

Fonte: Os autores.

Nos perfis de DSC, Figura 5, pode ser observado o pico endotérmico, a cerca de $250^{\circ} \mathrm{C}$, referente à fusão do PET (BANNACH et al, 2011). As amostras de compósitos com 5 e $7 \%$ de muscovita apresentaram ligeiro deslocamento do pico de fusão para temperaturas mais baixas e o $\mathrm{T}_{\text {onset }}$ também pareceu ocorrer em temperatura ligeiramente menor. Esta situação pode estar associada aos fatores explicados, para comprovar o comportamento apresentado pelo índice de fluidez pelas mesmas amostras. Além disso dados da literatura evidenciam que o polímero PET, geralmente, admite apenas baixos teores de cargas na sua estrutura, o que pode ser comprovado pelos resultados obtidos também nesse estudo (BANDA-CRUZ; FLORES-GALLARDO; RIVERA-ARMENTA, 2017; SIRELLI, 2008; LEITE et al., 2011).

Figura 5 - DSC para as amostras de PET puro e PET/Mica.

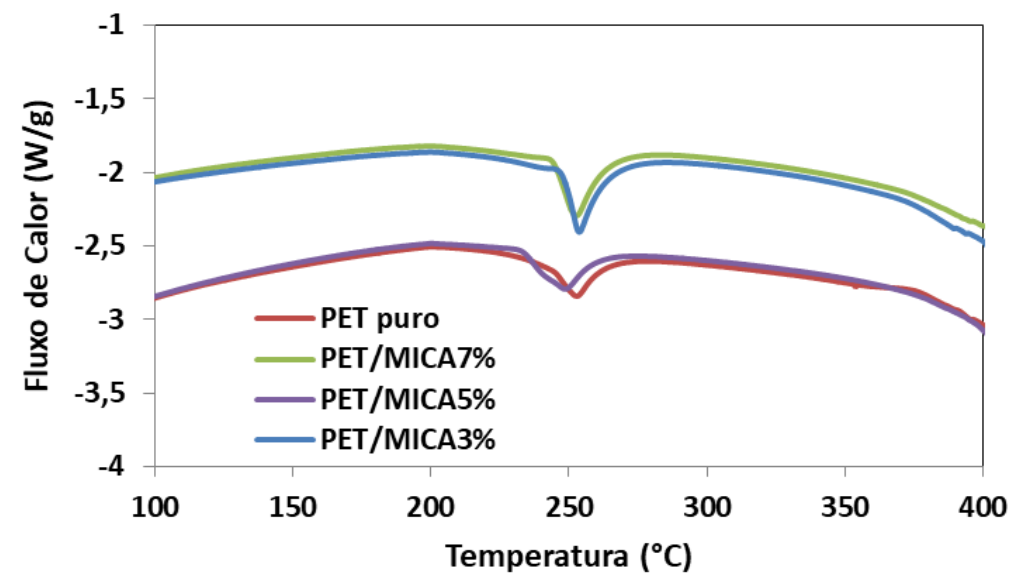

Fonte: Os autores.

As amostras também foram analisadas por microscopia óptica, num aumento de 
50X, oportunizando assim, observar a muscovita dispersa dentro da estrutura da matriz polimérica, como mostrado na Figura 6 que exibe as imagens obtidas para amostra de PET puro e para o compósito PET/Mica 7\%.

Figura 6 - Imagens de microscopia óptica do PET puro (a) e do compósito PET/Mica $7 \%$ (b). Aumento de 50X.
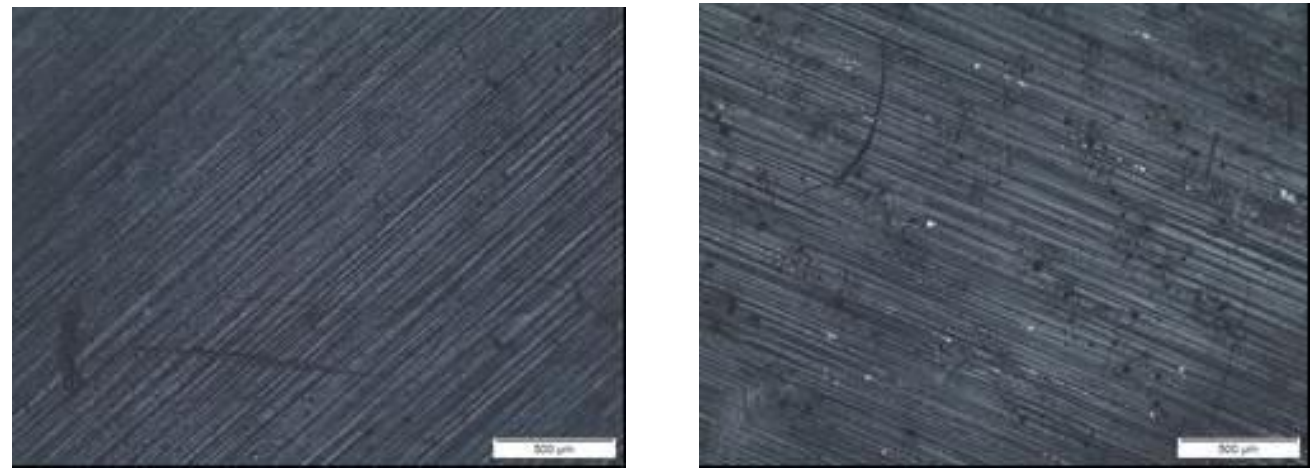

Fonte: Os autores.

\section{CONCLUSÕES}

Foi possível obter compósitos entre PET pós-consumo, oriundo de garrafas de bebidas, e mica do tipo muscovita. Os compósitos apresentaram tendência ao aumento da densidade e dureza com aumento do teor de mica, entretanto o índice de fluidez aumentou para teores de 5 e $7 \%$ de mica.

As análises térmicas revelaram que a carga não influenciou significativamente o perfil de decomposição térmica do polímero, porém a fusão sofreu ligeiro deslocamento para temperaturas mais baixas com os teores de 5 e $7 \%$ de mica.

Desta forma, confirmando dados já analisados para outros sistemas envolvendo PET e cargas inorgânicas, verificou-se que compósitos entre PET pós-consumo e mica, sem adição de compatibilizantes ou extensores de cadeia, parecem ser mais viáveis quando a concentração do mineral é inferior a 5\%.

\section{AGRADECIMENTOS}

Ao CETEM, na pessoa da pesquisadora Silvia França pelo fornecimento da mica e a FAPERJ pelo suporte financeiro.

\section{REFERÊNCIAS}

ALMEIDA, P. O. Compósitos de Madeira Plástica reforçada com Mica Muscovita. 2018. 77f. Dissertação (Mestrado Profissional em Ciência e Tecnologia dos Materiais). 
Centro Universitário Estadual da Zona Oeste - UEZO, Rio de Janeiro, 2018.

BALTAR, C. A. M. et al. Rochas e Minerais Industriais: usos e especificações, 2. ed., Rio de Janeiro: Editora CETEM/MCT, 2008.

BANDA-CRUZ, E. E.; FLORES-GALLARDO, S. G.; RIVERA-ARMENTA, J.L. Study of the dispersion of Cloisite $10 \mathrm{~A}$ in recycled polyethylene terephtalate by extrusion.

DYNA, v. 84, n. 200, p. 107-111, 2017.

BANNACH, G. et al. Efeitos da história térmica nas propriedades do polímero PET: um experimento para ensino de análise térmica. Química Nova, v. 34, n. 10, p. 18251829, 2011.

BIMESTRE, B. H.; SARON, C. Recuperação de propriedades de rejeitos de PET por meio de extrusão reativa com extensores de cadeia. Revista lberoamericana de Polímeros, v. 15, p. 252-260, 2014.

DU, P. et al. Effect of thermal activation on the tribological behaviors of muscovite particles as lubricant additives in lithium grease. Industrial Lubrication and Tribology, v. 70, n. 3, p. 538-543, 2018.

ESCÓCIO, V. A. et al. Influência da mica nas propriedades mecânicas e dinâmicomecânicas de composições de borracha natural. Polímeros, v. 13, n. 2, p. 130-134, 2003.

ESCÓCIO, V. A. et al. Caracterização térmica e dinâmico-mecânica de compósitos TPU/mica preparados em reômetro de torque. Polímeros, v. 21, n. 3, p. 240-245, 2011.

FERREIRA, C. T.; FONSECA, J. B.; SARON, C. Reciclagem de rejeitos de poli (tereftalato de etileno) (PET) e de poliamida (PA) por meio de extrusão reativa para a preparação de blendas. Polímeros, v. 21, n. 2, p. 118-122, 2011.

GANDARA, M.; GONÇALVES, A. R.; SARON, C. Compósitos de PET reciclado com fibra de cana-de-açucar tratada por explosão a vapor. Revista matéria, v. 22, n. 4, e11890, 2017.

LEITE, I. F. et al. Efeito de diferentes tipos de argilas e modificadores orgânicos na morfologia e propriedades térmicas dos nanocompósitos de PET. Polímeros, v. 21, n. 3, p. 195-203, 2011.

MANCINI, S. D.; BEZERRA, M. N.; ZANIN, M. Reciclagem de PET Advindo de garrafas de Refrigerante Pós-Consumo. Polímeros: Ciência e Tecnologia, v. 28, n.2, p. 68-75, 1998.

MONSORES, K. G. C. et al. Materiais Compósitos a base de PP e muscovita. Revista Iberoamericana de Polímeros, v. 18, n. 6, p. 301-309, 2017.

PEREIRA, A. P. S. et al. Processing and Characterization of PET Composites Reinforced With Geopolymer Concrete Waste. Materials Research, v. 20, n. 2, p. 411420, 2017.

RELOSI, N. et al.Effect of addition of clay minerals on the properties of epoxy/polyester powder coatings. Polímeros, v. 28, n. 4, p. 355-367, 2018. 
ROMÃO, W.; SPINACÉ, M. A. S.; DE PAOLI, M. A. Poli(tereftalato de etileno), PET: uma revisão sobre os processos de síntese, mecanismos de degradação e sua reciclagem. Polímeros, v. 19, n. 2, p. 121-132, 2009.

SANTOS, C.M.A. et al. Processo de reciclagem química de PET em meio alcalino: efeito da concentração do íon hidróxido, da cor do PET e do tempo de reação. Revista Matéria, v. 23, n. 4, p. 2018-2034, 2018.

SANTOS, S. F.; FRANÇA, S. C. A.; OGASAWARA, T. Method for grinding and delaminating muscovite, Mining Science and Technology, v. 21, n. 1, p. 7-10, 2011b.

SIRELLI, L. Compósitos de poli (tereftalato de etileno) e mica muscovita: preparação, caracterização e propriedades. 2008 Tese (Doutorado em Ciência e Tecnologia de Polímeros) - Universidade Federal do Rio de Janeiro, Rio de Janeiro, 2008.

TEÓFILO, E. T. et al. Falha por stress cracking em híbridos PET/argila. Polímeros, v. 24, n. 6, p. 694-702, 2014. 